Bull. Chem. Soc. Ethiop. 2020, 34(3), 533-541.

ISSN 1011-3924

(C) 2020 Chemical Society of Ethiopia and The Authors

Printed in Ethiopia

DOI: https://dx.doi.org/10.4314/bcse.v34i3.9

\title{
MIXED ISATIN WITH 3-(2-(ARYL)HYDRAZONO)ACETYLACETONE Mn(II), Co(II) AND Ni(II) COMPLEXES: ANTIBACTERIAL EVALUATION AND MOLECULAR PROPERTIES PREDICTION
}

\author{
Ashraf S. Hassan ${ }^{*}$ \\ Organometallic and Organometalloid Chemistry Department, National Research Centre, Dokki, \\ 12622, Cairo, Egypt
}

(Received March 18, 2020; Revised January 5, 2021; Accepted January 6, 2021)

\begin{abstract}
The metal complexes $\{\mathrm{Ni}$ (II), Co (II) and Mn (II) $\}$ of 3-(2-(aryl)hydrazono)acetylacetone with isatin were synthesized and screened for their in vitro antibacterial activity against four pathogenic microorganisms \{two Gram-positive and two Gram negative\}. The results of antibacterial activities revealed that all the metal complexes 1-9 exhibited moderate activities. Also, Lipinski's rule of five (RO5) of the mixed ligand metal complexes were calculated by Swiss ADME website.
\end{abstract}

KEY WORDS: Isatin, 3-(2-(Aryl)hydrazono)acetylacetone, Metal complexes, Antibacterial activities, Lipinski rules

\section{INTRODUCTION}

There is recently a growing interest in the field of synthesizing mixed ligand metal complexes due to various biological applications. In 2018, Sakr et al. have prepared $\mathrm{Zn}$ (II), Sn(II) and $\mathrm{Ce}(\mathrm{III})$ of gemifloxacin with glycine (A) as antibacterial agents [1]. Also, in 2017, Omar et al., have prepared the complexes of $\mathrm{Mn}(\mathrm{II}), \mathrm{Co}(\mathrm{II}), \mathrm{Ni}(\mathrm{II})$ and $\mathrm{Zn}(\mathrm{II})$ of Schiff base and 2,2'-bipyridine (B) for evaluation their antimicrobial and anticancer activities [2]. Literature survey revealed that isatin and its derivatives show a wide range of biological applications [3-5] such as 1-(1-(3-methylbenzyl)-2-oxoindolin-3-ylidene)-thiosemicarbazide (C) showed highly active against Mycobacterium bovis bacillus Calmette-Guerin [6] and 3-(benzylimino)-7chloroindolin-2-one (D) display significant cytotoxic activity against HeLa, SK-BR-3 and MCF7 cells [7]. Also, there are some isatin-based drugs, e.g. Sunitinib (Sutent $\left.{ }^{\circledR}\right)$ (E) acts as tyrosine kinase inhibitor [8]. Nintedanib (F) acts as triple angiokinase inhibitor [9] (Figure 1). In addition, arylhydrazono-1,3-diketone derivatives are useful synthons in the synthesis of a large number of biologically compounds [10-12].

In view of these facts and in continuation of our target [13-25], the metal complexes $\{\mathrm{Ni}(\mathrm{II})$, $\mathrm{Co}(\mathrm{II})$ and $\mathrm{Mn}(\mathrm{II})\}$ of 3-(2-(aryl)hydrazono)acetylacetone with isatin have been synthesized to evaluate their antibacterial activity against four pathogenic microorganisms two Gram-positive (Bacillus subtilis and Staphylococcus aureus) and two Gram negative (Escherichia coli and Pseudomonas aeruginosa) $\}$. Also, the study has been extended to calculate the physicochemical properties and drug-likeness (Lipinski's rule) of the mixed ligand metal complexes (Figure 2).

*Corresponding author. E-mail: ashraf_salmoon@yahoo.com

This work is licensed under the Creative Commons Attribution 4.0 International License 


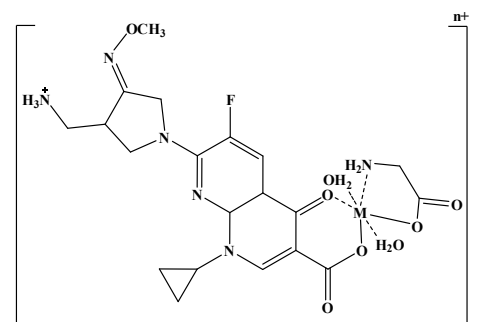

$\underset{\text { (A) }}{\text { Antibacterial agents }}$

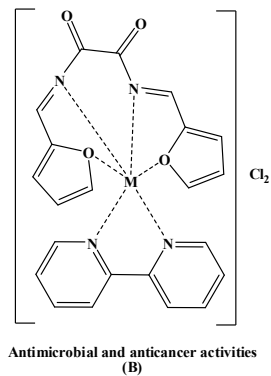

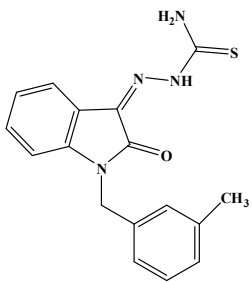

Antimycobacterial activity
(C)

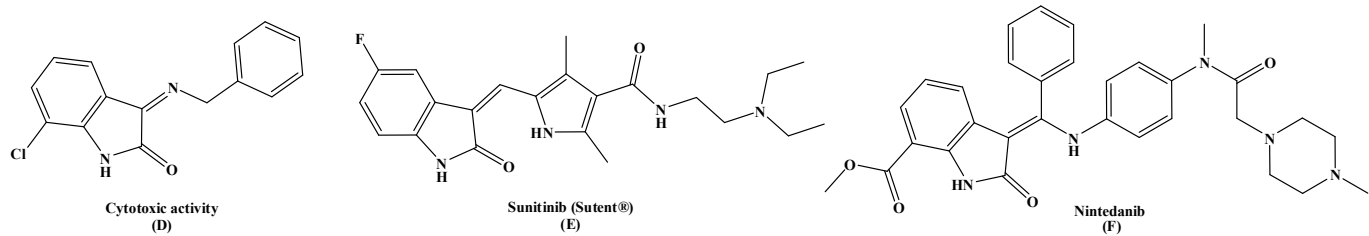

Figure 1. Structures of mixed complexes (A and $\mathbf{B})$ and isatin derivatives $\mathbf{C}-\mathbf{F}$.

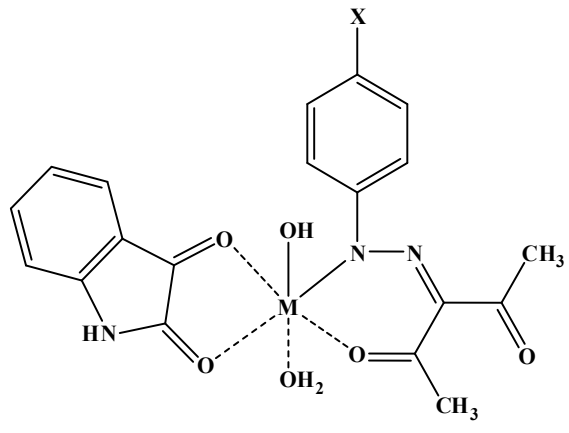

Antibacterial activity

physicochemical properties

Drug-likeness (Lipinski's rule)

Figure 2. Mixed ligand metal complexes and their studies.

\section{EXPERIMENTAL}

Preparation of 3-(arylhydrazono)acetylacetone $(\boldsymbol{H L})$

The 3-(arylhydrazono)acetylacetone derivatives (HL) were prepared by coupling acetylactone with different diazonium salts, where acetylactone $(0.01 \mathrm{~mol})$ was placed in a beaker containing ethanol $(50 \mathrm{~mL})$ and sodium acetate $(5 \mathrm{~g})$ then the diazonium salt $(0.01 \mathrm{~mol})$ was added with stirring. The reaction proceeded at $0-5{ }^{\circ} \mathrm{C}$ for $30 \mathrm{~min}$. The 3 -(arylhydrazono)acetylacetone derivatives were precipitated and collected by filtration, washed with cold deionized water and recrystallized from ethanol [26-28]. 
Mixed isatin with 3-(2-(aryl)hydrazono)acetylacetone $\mathrm{Mn}$ (II), $\mathrm{Co}(\mathrm{II})$ and $\mathrm{Ni(II)}$ complexes 535

3-(2-Phenylhydrazono)acetylacetone $\left(\boldsymbol{H} \boldsymbol{L}^{1}\right)$. Yellow needles; yield: $86 \%$; m.p. $86-87^{\circ} \mathrm{C}$; anal. calcd. (\%) for $\mathrm{C}_{11} \mathrm{H}_{12} \mathrm{~N}_{2} \mathrm{O}_{2}$ (204.23): $\mathrm{C}, 64.69 ; \mathrm{H}, 5.92 ; \mathrm{N}, 13.72$. Found: $\mathrm{C}, 64.50 ; \mathrm{H}, 6.10 ; \mathrm{N}$, $13.65 \%$; IR (KBr) v $v_{\max } / \mathrm{cm}^{-1} 3430-3092(\underline{\mathrm{N}-\mathrm{H}} \ldots \mathrm{O}=\mathrm{C}$, hydrogen bonded), $1673(\mathrm{C}=\mathrm{O}$, free), 1623 $\left(\mathrm{C}=\mathrm{O}\right.$, hydrogen bonded), $1593(\mathrm{C}=\mathrm{N}), 1518(\mathrm{~N}-\mathrm{H}) ;{ }^{1} \mathrm{H}$ NMR $\left(\right.$ DMSO-d $\left._{6}, \delta \mathrm{ppm}\right) 2.36(\mathrm{~s}, 3 \mathrm{H}$, $\mathrm{CH}_{3} \mathrm{CO}$ free), 2.43 (s, $3 \mathrm{H}, \mathrm{CH}_{3} \mathrm{CO}$ hydrogen bonded), 7.16 (t, $1 \mathrm{H}$, aromatic), 7.27 (t, $2 \mathrm{H}$, aromatic), 7.52 (d, $2 \mathrm{H}, J=8.5 \mathrm{~Hz}$, aromatic), 14.02 (s, $1 \mathrm{H}, \mathrm{NH}, \mathrm{D}_{2} \mathrm{O}$ exchangeable).

3-(2-(4-Chlorophenyl)hydrazono)acetylacetone $\left(\boldsymbol{H L}^{2}\right)$. Yellow needles; yield: 86\%; m.p. 147$148{ }^{\circ} \mathrm{C}$; anal. calcd. (\%) for $\mathrm{C}_{11} \mathrm{H}_{11} \mathrm{ClN}_{2} \mathrm{O}_{2}$ (238.67): C, 55.36; H, 4.65; N, 11.74. Found: $\mathrm{C}$, $55.50 ; \mathrm{H}, 4.52 ; \mathrm{N}, 11.80 \%$; IR $(\mathrm{KBr}) v_{\max } / \mathrm{cm}^{-1} 3432-3094(\underline{\mathrm{N}-\mathrm{H}} \ldots \mathrm{O}=\mathrm{C}$, hydrogen bonded), $1667\left(\mathrm{C}=\mathrm{O}\right.$, free), $1625(\mathrm{C}=\mathrm{O}$, hydrogen bonded $), 1589(\mathrm{C}=\mathrm{N}), 1519(\mathrm{~N}-\mathrm{H}) ;{ }^{1} \mathrm{H}$ NMR (DMSO-d $\left.{ }_{6}, \delta \mathrm{ppm}\right) 2.37$ (s, $3 \mathrm{H}, \mathrm{CH}_{3} \mathrm{CO}$ free), 2.42 (s, 3H, $\mathrm{CH}_{3} \mathrm{CO}$ hydrogen bonded), 7.43 (d, $2 \mathrm{H}, J=8.4 \mathrm{~Hz}$, aromatic), $7.57\left(\mathrm{~d}, 2 \mathrm{H}, J=8.4 \mathrm{~Hz}\right.$, aromatic), $13.81\left(\mathrm{~s}, 1 \mathrm{H}, \mathrm{NH}, \mathrm{D}_{2} \mathrm{O}\right.$ exchangeable).

3-(2-(4-Bromophenyl)hydrazono)acetylacetone $\left(\boldsymbol{H L}^{3}\right)$. Yellow needles; yield 86\%; m.p. 141$142{ }^{\circ} \mathrm{C}$; anal. calcd. (\%) for $\mathrm{C}_{11} \mathrm{H}_{11} \mathrm{BrN}_{2} \mathrm{O}_{2}$ (283.12): $\mathrm{C}, 46.66 ; \mathrm{H}, 3.92 ; \mathrm{N}, 9.89$. Found: $\mathrm{C}, 46.50$; $\mathrm{H}, 4.10 ; \mathrm{N}, 10.00 \%$; IR $(\mathrm{KBr}) v_{\max } / \mathrm{cm}^{-1} 3422-3073(\underline{\mathrm{N}-\mathrm{H}} \ldots \mathrm{O}=\mathrm{C}$, hydrogen bonded), 1667 $\left(\mathrm{C}=\mathrm{O}\right.$, free), $1623(\mathrm{C}=\mathrm{O}$, hydrogen bonded $), 1585(\mathrm{C}=\mathrm{N}), 1513(\mathrm{~N}-\mathrm{H}) ;{ }^{1} \mathrm{H}$ NMR $\left(\mathrm{DMSO}_{6}, \delta\right.$ ppm) 2.37 (s, 3H, $\mathrm{CH}_{3} \mathrm{CO}$ free), $2.42\left(\mathrm{~s}, 3 \mathrm{H}, \mathrm{CH}_{3} \mathrm{CO}\right.$ hydrogen bonded), 7.50 (d, $2 \mathrm{H}, J=9.15$ $\mathrm{Hz}$, aromatic), $7.56\left(\mathrm{~d}, 2 \mathrm{H}, J=9.15 \mathrm{~Hz}\right.$, aromatic), 13.75 (s, $1 \mathrm{H}, \mathrm{NH}, \mathrm{D}_{2} \mathrm{O}$ exchangeable).

Isatin $\left(\boldsymbol{L}^{\prime}\right)$. IR $(\mathrm{KBr}) v_{\max } / \mathrm{cm}^{-1} 3191(\mathrm{~N}-\mathrm{H}), 1745(\mathrm{C}=\mathrm{O}), 1731(\mathrm{C}=\mathrm{O}) ;{ }^{1} \mathrm{H}$ NMR $\left(\mathrm{DMSO}^{-} \mathrm{d}_{6}, \delta\right.$ ppm) $6.88(\mathrm{~d}, 1 \mathrm{H}, J=7.7 \mathrm{~Hz}$, aromatic), $7.03(\mathrm{t}, 1 \mathrm{H}$, aromatic), $7.46(\mathrm{~d}, 1 \mathrm{H}, J=7.7 \mathrm{~Hz}$, aromatic), 7.54 (t, $1 \mathrm{H}$, aromatic), $10.98\left(\mathrm{~s}, 1 \mathrm{H}, \mathrm{NH}, \mathrm{D}_{2} \mathrm{O}\right.$ exchangeable).

\section{Preparation of the complexes 1-9}

A solution of the metal chloride $(3.4 \mathrm{mmol})$ in a minimum amount of water was added to a hot solution of the mixed ligand in methanol ( $3.4 \mathrm{mmol}$ each). A clear solution was obtained. The $\mathrm{pH}$ was raised from 6.0 to 8.0 with dilute $\mathrm{NaOH}$ solution. The mixture was refluxed with stirring for 6 hours. The formed complex was filtered off, washed several times with hot methanol and dried under reduced pressure [29].

$\left[\mathrm{NiL}^{\prime} \mathrm{L}^{1}(\mathrm{OH})\left(\mathrm{H}_{2} \mathrm{O}\right)\right]$ (1). Greenish yellow; yield 50\%; m.p. $215-218{ }^{\circ} \mathrm{C}$; anal. calcd. (\%) for $\mathrm{C}_{19} \mathrm{H}_{19} \mathrm{~N}_{3} \mathrm{NiO}_{6}$ (444.06): C, 51.39; H, 4.31; N, 9.46. Found: $\mathrm{C}, 51.50 ; \mathrm{H}, 4.25 ; \mathrm{N}, 9.55 \%$; IR $(\mathrm{KBr}) v_{\max } / \mathrm{cm}^{-1} 3522(\mathrm{O}-\mathrm{H}), 3181\left(\mathrm{~N}-\mathrm{H}, \mathrm{L}^{\prime}\right), 1717\left(\mathrm{C}=\mathrm{O}, \mathrm{L}^{\prime}\right), 1709\left(\mathrm{C}=\mathrm{O}, \mathrm{L}^{\prime}\right), 1667(\mathrm{C}=\mathrm{O}$ free, $\left.\mathrm{L}^{1}\right), 1600\left(\mathrm{C}=\mathrm{O}\right.$ coordinated, $\left.\mathrm{L}^{1}\right), 1590\left(\mathrm{C}=\mathrm{N}, \mathrm{L}^{1}\right), 583(\mathrm{Ni}-\mathrm{N}), 493(\mathrm{Ni}-\mathrm{O}) ;{ }^{1} \mathrm{H}$ NMR (DMSO$\left.\mathrm{d}_{6}, \delta \mathrm{ppm}\right) 2.34\left(\mathrm{~s}, 3 \mathrm{H}, \mathrm{CH}_{3} \mathrm{CO}\right.$ free, $\left.\mathrm{L}^{1}\right), 2.64\left(\mathrm{~s}, 3 \mathrm{H}, \mathrm{CH}_{3} \mathrm{CO}\right.$ coordinated, $\left.\mathrm{L}^{1}\right), 6.76-7.58(\mathrm{~m}$, $9 \mathrm{H}$, aromatic, $\mathrm{L}^{\prime}$ and $\left.\mathrm{L}^{1}\right), 10.20\left(\mathrm{~s}, 1 \mathrm{H}, \mathrm{NH}, \mathrm{D}_{2} \mathrm{O}\right.$ exchangeable, $\left.\mathrm{L}^{\prime}\right)$; UV-Vis. $\lambda=963,576,420$ $\mathrm{nm}$; magnetic moment $\left(\mu_{\text {eff }}\right.$ B.M.): 3.17 ; molar conductivity $\left(\Omega^{-1} \mathrm{~cm}^{2} \mathrm{~mol}^{-1}\right): 11.6$.

[CoL' $\left.\mathrm{L}^{1}(\mathrm{OH})\left(\mathrm{H}_{2} \mathrm{O}\right)\right]$ (2). Dark green; yield 53\%; m.p. 209-211 ${ }^{\circ} \mathrm{C}$; anal. calcd. (\%) for $\mathrm{C}_{19} \mathrm{H}_{19} \mathrm{CoN}_{3} \mathrm{O}_{6}$ (444.30): C, 51.36; H, 4.31; N, 9.46. Found: C, 51.55; H, 4.25; N, 9.55\%; IR $(\mathrm{KBr}) v_{\max } / \mathrm{cm}^{-1} 3580(\mathrm{O}-\mathrm{H}), 3183\left(\mathrm{~N}-\mathrm{H}, \mathrm{L}^{\prime}\right), 1715\left(\mathrm{C}=\mathrm{O}, \mathrm{L}^{\prime}\right), 1707\left(\mathrm{C}=\mathrm{O}, \mathrm{L}^{\prime}\right), 1669(\mathrm{C}=\mathrm{O}$ free, $\left.\mathrm{L}^{1}\right), 1602\left(\mathrm{C}=\mathrm{O}\right.$ coordinated, $\left.\mathrm{L}^{1}\right), 1591\left(\mathrm{C}=\mathrm{N}, \mathrm{L}^{1}\right), 584(\mathrm{Co}-\mathrm{N}), 491(\mathrm{Co}-\mathrm{O})$; UV-Vis. $\lambda=654$, $540 \mathrm{~nm}$; magnetic moment $\left(\mu_{\text {eff }}\right.$ B.M. $)$ : 4.92; molar conductivity $\left(\Omega^{-1} \mathrm{~cm}^{2} \mathrm{~mol}^{-1}\right): 12.8$.

[MnL'L $\left.\mathrm{L}^{l}(\mathrm{OH})\left(\mathrm{H}_{2} \mathrm{O}\right)\right]$ (3). Green; yield 45\%; m.p. $210-212{ }^{\circ} \mathrm{C}$; anal. calcd. (\%) for $\mathrm{C}_{19} \mathrm{H}_{19} \mathrm{MnN}_{3} \mathrm{O}_{6}$ (440.31): C, 51.83; H, 4.35; N, 9.54. Found: C, 51.70; H, 4.40; N, 9.47\%; IR $(\mathrm{KBr}) v_{\max } / \mathrm{cm}^{-1} 3544(\mathrm{O}-\mathrm{H}), 3182\left(\mathrm{~N}-\mathrm{H}, \mathrm{L}^{\prime}\right), 1719\left(\mathrm{C}=\mathrm{O}, \mathrm{L}^{\prime}\right), 1711\left(\mathrm{C}=\mathrm{O}, \mathrm{L}^{\prime}\right), 1661(\mathrm{C}=\mathrm{O}$ free, 
$\left.\mathrm{L}^{1}\right), 1605\left(\mathrm{C}=\mathrm{O}\right.$ coordinated, $\left.\mathrm{L}^{1}\right), 1590\left(\mathrm{C}=\mathrm{N}, \mathrm{L}^{1}\right), 540(\mathrm{Mn}-\mathrm{N}), 487(\mathrm{Mn}-\mathrm{O})$; UV-Vis. $\lambda=752$, $618,520 \mathrm{~nm}$; magnetic moment $\left(\mu_{e f f}\right.$ B.M. $): 5.82$; molar conductivity $\left(\Omega^{-1} \mathrm{~cm}^{2} \mathrm{~mol}^{-1}\right): 15.4$.

$\left[\mathrm{NiL}^{\prime} \mathrm{L}^{2}(\mathrm{OH})\left(\mathrm{H}_{2} \mathrm{O}\right)\right]$ (4). Greenish yellow; yield $60 \%$; m.p. $217-220{ }^{\circ} \mathrm{C}$; anal. calcd. (\%) for $\mathrm{C}_{19} \mathrm{H}_{18} \mathrm{ClN}_{3} \mathrm{NiO}_{6}$ (478.51): C, 47.69; H, 3.79; N, 8.78. Found: $\mathrm{C}, 47.50 ; \mathrm{H}, 3.70 ; \mathrm{N}, 8.70 \%$; IR $(\mathrm{KBr}) v_{\max } / \mathrm{cm}^{-1} 3565(\mathrm{O}-\mathrm{H}), 3180\left(\mathrm{~N}-\mathrm{H}, \mathrm{L}^{\prime}\right), 1715\left(\mathrm{C}=\mathrm{O}, \mathrm{L}^{\prime}\right), 1707\left(\mathrm{C}=\mathrm{O}, \mathrm{L}^{\prime}\right), 1661(\mathrm{C}=\mathrm{O}$ free, $\left.\mathrm{L}^{2}\right), 1605\left(\mathrm{C}=\mathrm{O}\right.$ coordinated, $\left.\mathrm{L}^{2}\right), 1585\left(\mathrm{C}=\mathrm{N}, \mathrm{L}^{2}\right), 579(\mathrm{Ni}-\mathrm{N}), 448(\mathrm{Ni}-\mathrm{O}) ;{ }^{1} \mathrm{H}$ NMR (DMSO$\left.\mathrm{d}_{6}, \delta \mathrm{ppm}\right) 2.30\left(\mathrm{~s}, 3 \mathrm{H}, \mathrm{CH}_{3} \mathrm{CO}\right.$ free, $\left.\mathrm{L}^{2}\right), 2.60\left(\mathrm{~s}, 3 \mathrm{H}, \mathrm{CH}_{3} \mathrm{CO}\right.$ coordinated, $\left.\mathrm{L}^{2}\right), 6.74-7.60(\mathrm{~m}$, $8 \mathrm{H}$, aromatic, $\mathrm{L}^{\prime}$ and $\left.\mathrm{L}^{2}\right), 10.20\left(\mathrm{~s}, 1 \mathrm{H}, \mathrm{NH}, \mathrm{D}_{2} \mathrm{O}\right.$ exchangeable, $\left.\mathrm{L}^{\prime}\right)$; UV-Vis. $\lambda=988,581,422$ $\mathrm{nm}$; magnetic moment $\left(\mu_{\text {eff }} /\right.$ B.M. $): 3.20$; molar conductivity $\left(\Omega^{-1} \mathrm{~cm}^{2} \mathrm{~mol}^{-1}\right): 20.5$.

$\left[\mathrm{CoL}^{\prime} \mathrm{L}^{2}(\mathrm{OH})\left(\mathrm{H}_{2} \mathrm{O}\right)\right]$ (5). Dark green; yield 58\%; m.p. 207-209 ${ }^{\circ} \mathrm{C}$; anal. calcd. (\%) for $\mathrm{C}_{19} \mathrm{H}_{18} \mathrm{ClCoN}_{3} \mathrm{O}_{6}$ (478.75): C, 47.67; H, 3.79; N, 8.78. Found: C, 47.50; H, 3.85; N, 8.70\%; IR $(\mathrm{KBr}) v_{\max } / \mathrm{cm}^{-1} 3553(\mathrm{O}-\mathrm{H}), 3186\left(\mathrm{~N}-\mathrm{H}, \mathrm{L}^{\prime}\right), 1716\left(\mathrm{C}=\mathrm{O}, \mathrm{L}^{\prime}\right), 1708\left(\mathrm{C}=\mathrm{O}, \mathrm{L}^{\prime}\right), 1662(\mathrm{C}=\mathrm{O}$ free, $\left.\mathrm{L}^{2}\right), 1602\left(\mathrm{C}=\mathrm{O}\right.$ coordinated, $\left.\mathrm{L}^{2}\right), 1584\left(\mathrm{C}=\mathrm{N}, \mathrm{L}^{2}\right), 572(\mathrm{Co}-\mathrm{N}), 438(\mathrm{Co}-\mathrm{O})$; UV-Vis. $\lambda=661$, $548 \mathrm{~nm}$; magnetic moment $\left(\mu_{\text {eff }}\right.$ B.M. $)$ : 4.94 ; molar conductivity $\left(\Omega^{-1} \mathrm{~cm}^{2} \mathrm{~mol}^{-1}\right): 22.1$.

$\left[\mathrm{MnL}^{\prime} \mathrm{L}^{2}(\mathrm{OH})\left(\mathrm{H}_{2} \mathrm{O}\right)\right]$ (6). Greenish yellow; yield 55\%; m.p. $216-219{ }^{\circ} \mathrm{C}$; anal. calcd. (\%) for $\mathrm{C}_{19} \mathrm{H}_{18} \mathrm{ClMnN}_{3} \mathrm{O}_{6}$ (474.75): C, 48.07; H, 3.82; N, 8.85. Found: C, 48.20; H, 3.75; N, 8.80\%; IR $(\mathrm{KBr}) v_{\max } / \mathrm{cm}^{-1} 3550(\mathrm{O}-\mathrm{H}), 3183\left(\mathrm{~N}-\mathrm{H}, \mathrm{L}^{\prime}\right), 1718\left(\mathrm{C}=\mathrm{O}, \mathrm{L}^{\prime}\right), 1710\left(\mathrm{C}=\mathrm{O}, \mathrm{L}^{\prime}\right), 1661(\mathrm{C}=\mathrm{O}$ free, $\left.\mathrm{L}^{2}\right), 1605\left(\mathrm{C}=\mathrm{O}\right.$ coordinated, $\left.\mathrm{L}^{2}\right), 1580\left(\mathrm{C}=\mathrm{N}, \mathrm{L}^{2}\right), 569(\mathrm{Mn}-\mathrm{N}), 444(\mathrm{Mn}-\mathrm{O}) ;{ }^{1} \mathrm{H} \mathrm{NMR}$ $\left(\mathrm{DMSO}_{6}, \delta \mathrm{ppm}\right) 2.33\left(\mathrm{~s}, 3 \mathrm{H}, \mathrm{CH}_{3} \mathrm{CO}\right.$ free, $\left.\mathrm{L}^{2}\right), 2.61\left(\mathrm{~s}, 3 \mathrm{H}, \mathrm{CH}_{3} \mathrm{CO}\right.$ coordinated, $\left.\mathrm{L}^{2}\right), 6.74-$ $7.58\left(\mathrm{~m}, 8 \mathrm{H}\right.$, aromatic, $\mathrm{L}^{\prime}$ and $\left.\mathrm{L}^{2}\right), 10.21\left(\mathrm{~s}, 1 \mathrm{H}, \mathrm{NH}, \mathrm{D}_{2} \mathrm{O}\right.$ exchangeable, $\left.\mathrm{L}^{\prime}\right)$; UV-Vis. $\lambda=758$, $622,526 \mathrm{~nm}$; magnetic moment ( $\mu_{\text {eff }}$ B.M.): 5.90; molar conductivity $\left(\Omega^{-1} \mathrm{~cm}^{2} \mathrm{~mol}^{-1}\right): 19.8$.

$\left[\mathrm{NiL}^{\prime} \mathrm{L}^{3}(\mathrm{OH})\left(\mathrm{H}_{2} \mathrm{O}\right)\right](7)$. Greenish yellow; yield 53\%; m.p. $223-226^{\circ} \mathrm{C}$; anal. calcd. (\%) for $\mathrm{C}_{19} \mathrm{H}_{18} \mathrm{BrN}_{3} \mathrm{NiO}_{6}$ (522.96): C, 43.64; H, 3.47; N, 8.04. Found: $\mathrm{C}, 43.50 ; \mathrm{H}, 3.55 ; \mathrm{N}, 8.00 \%$; IR $(\mathrm{KBr}) v_{\max } / \mathrm{cm}^{-1} 3575(\mathrm{O}-\mathrm{H}), 3184\left(\mathrm{~N}-\mathrm{H}, \mathrm{L}^{\prime}\right), 1722\left(\mathrm{C}=\mathrm{O}, \mathrm{L}^{\prime}\right), 1711\left(\mathrm{C}=\mathrm{O}, \mathrm{L}^{\prime}\right), 1660(\mathrm{C}=\mathrm{O}$ free, $\left.\mathrm{L}^{3}\right), 1600\left(\mathrm{C}=\mathrm{O}\right.$ coordinated, $\left.\mathrm{L}^{3}\right), 1580\left(\mathrm{C}=\mathrm{N}, \mathrm{L}^{3}\right), 578(\mathrm{Ni}-\mathrm{N}), 447(\mathrm{Ni}-\mathrm{O}) ;{ }^{1} \mathrm{H}$ NMR (DMSO$\left.\mathrm{d}_{6}, \delta \mathrm{ppm}\right) 2.33\left(\mathrm{~s}, 3 \mathrm{H}, \mathrm{CH}_{3} \mathrm{CO}\right.$ free, $\left.\mathrm{L}^{3}\right), 2.62\left(\mathrm{~s}, 3 \mathrm{H}, \mathrm{CH}_{3} \mathrm{CO}\right.$ coordinated, $\left.\mathrm{L}^{3}\right), 6.75-7.57(\mathrm{~m}$, $8 \mathrm{H}$, aromatic, $\mathrm{L}^{\prime}$ and $\left.\mathrm{L}^{3}\right), 10.20\left(\mathrm{~s}, 1 \mathrm{H}, \mathrm{NH}, \mathrm{D}_{2} \mathrm{O}\right.$ exchangeable, $\left.\mathrm{L}^{\prime}\right)$; $\mathrm{UV}-\mathrm{Vis}$. $\lambda=979,572,420$ $\mathrm{nm}$; magnetic moment $\left(\mu_{\text {eff }} /\right.$ B.M. $): 3.19$; molar conductivity $\left(\Omega^{-1} \mathrm{~cm}^{2} \mathrm{~mol}^{-1}\right): 18.7$.

$\left[\mathrm{CoL}^{\prime} \mathrm{L}^{3}(\mathrm{OH})\left(\mathrm{H}_{2} \mathrm{O}\right)\right]$ (8). Greenish yellow; yield 51\%; m.p. $212-215{ }^{\circ} \mathrm{C}$; anal. calcd. (\%) for $\mathrm{C}_{19} \mathrm{H}_{18} \mathrm{BrCoN}_{3} \mathrm{O}_{6}$ (523.20): C, 43.62; H, 3.47; N, 8.03. Found: C, 43.80; H, 3.40; N, 8.10\%; IR $(\mathrm{KBr}) v_{\max } / \mathrm{cm}^{-1} 3535(\mathrm{O}-\mathrm{H}), 3184\left(\mathrm{~N}-\mathrm{H}, \mathrm{L}^{\prime}\right), 1719\left(\mathrm{C}=\mathrm{O}, \mathrm{L}^{\prime}\right), 1707\left(\mathrm{C}=\mathrm{O}, \mathrm{L}^{\prime}\right), 1662(\mathrm{C}=\mathrm{O}$ free, $\left.\mathrm{L}^{3}\right), 1601\left(\mathrm{C}=\mathrm{O}\right.$ coordinated, $\left.\mathrm{L}^{3}\right), 1582\left(\mathrm{C}=\mathrm{N}, \mathrm{L}^{3}\right), 584(\mathrm{Co}-\mathrm{N}), 481(\mathrm{Co}-\mathrm{O})$; UV-Vis. $\lambda=659$, $544 \mathrm{~nm}$; magnetic moment ( $\mu_{\text {eff }}$ B.M.): 4.87 ; molar conductivity $\left(\Omega^{-1} \mathrm{~cm}^{2} \mathrm{~mol}^{-1}\right): 16.9$.

$\left[M n L^{\prime} L^{3}(\mathrm{OH})\left(\mathrm{H}_{2} \mathrm{O}\right)\right]$ (9). Green; yield 45\%; m.p. 215-218 ${ }^{\circ} \mathrm{C}$; anal. calcd. (\%) for $\mathrm{C}_{19} \mathrm{H}_{18} \mathrm{BrMnN}_{3} \mathrm{O}_{6}$, (519.20): C, 43.95; H, 3.49; N, 8.09. Found: C, 44.10; H, 3.40; N, 8.15\%; IR $(\mathrm{KBr}) v_{\max } / \mathrm{cm}^{-1} 3545(\mathrm{O}-\mathrm{H}), 3182\left(\mathrm{~N}-\mathrm{H}, \mathrm{L}^{\prime}\right), 1721\left(\mathrm{C}=\mathrm{O}, \mathrm{L}^{\prime}\right), 1712\left(\mathrm{C}=\mathrm{O}, \mathrm{L}^{\prime}\right), 1661(\mathrm{C}=\mathrm{O}$ free, $\left.\mathrm{L}^{3}\right), 1602\left(\mathrm{C}=\mathrm{O}\right.$ coordinated, $\left.\mathrm{L}^{3}\right), 1582\left(\mathrm{C}=\mathrm{N}, \mathrm{L}^{3}\right), 571(\mathrm{Mn}-\mathrm{N}), 472(\mathrm{Mn}-\mathrm{O}) ;{ }^{1} \mathrm{H}$ NMR $\left(\mathrm{DMSO}_{6}, \delta \mathrm{ppm}\right) 2.32\left(\mathrm{~s}, 3 \mathrm{H}, \mathrm{CH}_{3} \mathrm{CO}\right.$ free, $\left.\mathrm{L}^{3}\right), 2.60\left(\mathrm{~s}, 3 \mathrm{H}, \mathrm{CH}_{3} \mathrm{CO}\right.$ coordinated, $\left.\mathrm{L}^{3}\right), 6.74-$ $7.58\left(\mathrm{~m}, 8 \mathrm{H}\right.$, aromatic, $\mathrm{L}^{\prime}$ and $\left.\mathrm{L}^{3}\right), 10.21\left(\mathrm{~s}, 1 \mathrm{H}, \mathrm{NH}, \mathrm{D}_{2} \mathrm{O}\right.$ exchangeable, $\left.\mathrm{L}^{\prime}\right)$; UV-Vis. $\lambda=755$, $621,524 \mathrm{~nm}$; magnetic moment $\left(\mu_{e f f}\right.$ B.M.): 5.87 ; molar conductivity $\left(\Omega^{-1} \mathrm{~cm}^{2} \mathrm{~mol}^{-1}\right): 17.3$.

\section{Antibacterial activities}

$\mathrm{Mn}(\mathrm{II}), \mathrm{Co}(\mathrm{II})$ and $\mathrm{Ni}$ (II) complexes of mixed isatin with 3-(2-(aryl)hydrazono)acetylacetone 19 were screened in vitro for their antibacterial activities against two Gram-positive bacteria 
species (Bacillus subtilis, Staphylococcus aureus) and two-Gram negative bacteria species (Escherichia coli, Pseudomonas aeruginosa) using a modified Kirby-Bauer disc diffusion method [30]. The bacteria were maintained on Meuller-Hinton agar. DMSO showed no inhibition zone. The agar media were incubated at $35-37^{\circ} \mathrm{C}$ for $24-48$ hours for bacteria. The diameter of inhibition zone in millimeter $(\mathrm{mm})$ was measured. Tetracycline is used as a reference for antibacterial activities.

\section{RESULTS AND DISCUSSION}

\section{Chemistry}

3-(Arylhydrazono)acetylacetone (HL) [26-28] is prepared via coupling between diazotized aromatic amine derivatives and acetylacetone in a 1:1 molar ratio according to Scheme 1.

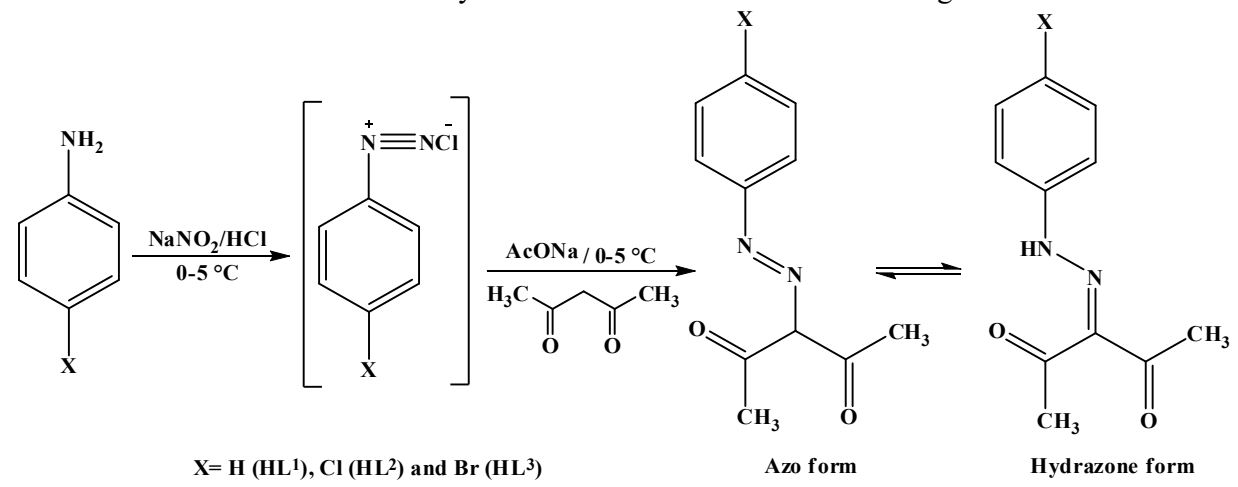

Scheme 1

3-(Arylhydrazono)acetylacetone (HL) and isatin $\left(\mathbf{L}^{\prime}\right)$ were reacted with hydrated metal(II) chloride in equimolar ratios (1:1:1) for formation the complexes 1-9 having the general structural formula [ML'L $\left.(\mathrm{OH})\left(\mathrm{H}_{2} \mathrm{O}\right)\right]$ (Scheme 2) [29].

\section{In vitro antibacterial activities}

The antibacterial activities of the mixed ligand metal complexes 1-9 against two-Gram negative bacteria species (Escherichia coli and Pseudomonas aeruginosa) and two Gram-positive bacteria species (Bacillus subtilis and Staphylococcus aureus) using a modified Kirby-Bauer disc diffusion method [30] are represented in Table 1. The results revealed that the metal complexes exhibited moderate activities.

In case of $E$. coli (G-), all the mixed metal complexes exhibited moderate activities in the range of inhibition zone diameter from 9 to $13 \mathrm{~mm}$. The two complexes 3 and 4 (IZ = $13 \mathrm{~mm}$ ) were more active than all the other tested complexes. In case of $P$. aeruginosa (G-), the three mixed metal complexes $\mathbf{3}, 5$ and $\mathbf{8}(\mathrm{IZ}=13 \mathrm{~mm})$ were more active than all the other tested complexes. By testing the mixed metal complexes against two Gram-positive bacteria species (Bacillus subtilis and Staphylococcus aureus), complex 6 was more potent $(\mathrm{IZ}=13 \mathrm{~mm}$ ) than the other tested complexes.

In general, all the mixed metal complexes 1-9 exhibit moderate activities against the four bacteria species used in this study.In particular, the two complexes $\left[\mathrm{MnL}^{\prime} \mathrm{L}^{1}(\mathrm{OH})\left(\mathrm{H}_{2} \mathrm{O}\right)\right](\mathbf{3})$ and $\left[\mathrm{MnL}^{\prime} \mathrm{L}^{2}(\mathrm{OH})\left(\mathrm{H}_{2} \mathrm{O}\right)\right](6)$ which having $\mathrm{Mn}(\mathrm{II})$ metal in their structures. May be the introduction 
the $\mathrm{Mn}$ (II) metal in the structure increasing the activities. Therefore, in the future, we will modify the mixed metal complexes to obtain more active antibacterial agents.

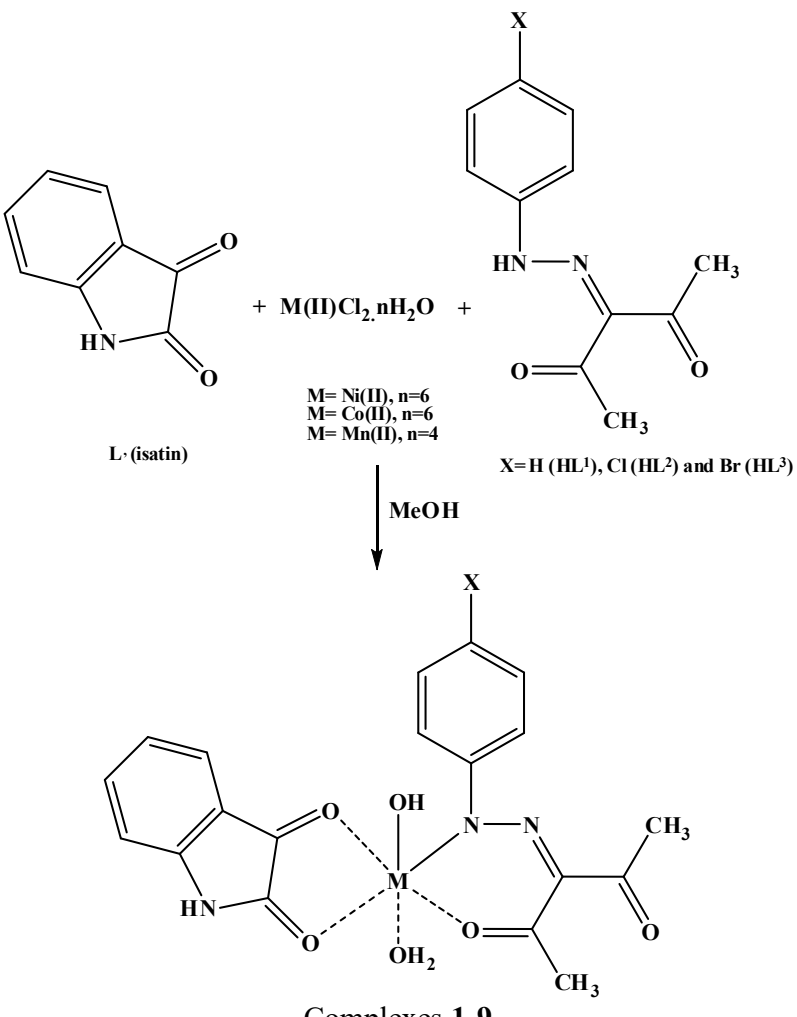

Complexes 1-9

\begin{tabular}{|c|c|c|c|c|c|}
\hline Complexes & $\mathrm{M}(\mathrm{II})$ & $\mathrm{X}$ & Complexes & $\mathrm{M}(\mathrm{II})$ & $\mathrm{X}$ \\
\hline $\mathbf{1}$ & $\mathrm{Ni}$ & $\mathrm{H}$ & $\mathbf{6}$ & $\mathrm{Mn}$ & $\mathrm{Cl}$ \\
\hline $\mathbf{2}$ & $\mathrm{Co}$ & $\mathrm{H}$ & $\mathbf{7}$ & $\mathrm{Ni}$ & $\mathrm{Br}$ \\
\hline $\mathbf{3}$ & $\mathrm{Mn}$ & $\mathrm{H}$ & $\mathbf{8}$ & $\mathrm{Co}$ & $\mathrm{Br}$ \\
\hline $\mathbf{4}$ & $\mathrm{Ni}$ & $\mathrm{Cl}$ & $\mathbf{9}$ & $\mathrm{Mn}$ & $\mathrm{Br}$ \\
\hline $\mathbf{5}$ & $\mathrm{Co}$ & $\mathrm{Cl}$ & & & \\
\hline
\end{tabular}

Scheme 2

Lipinski's rule of five (RO5) of the mixed ligand metal complexes

Lipinski's rule of five (RO5), is a rule of thumb to evaluate drug likeness or determine if a chemical compound with a certain pharmacological or biological activity has chemical properties and physical properties that would make it a likely orally active drug in humans. The rule was formulated by Christopher A. Lipinski in 1997, based on the observation that most orally administered drugs are relatively small and moderately lipophilic molecules [31-32].

The molecular weight $(\mathrm{MW} \leq 500)$, lipophilicity $(M \log P \leq 4.15)$, the number of hydrogen bond acceptors $(\mathrm{nHBA} \leq 10)$ and donors $(\mathrm{nHBD} \leq 5)$ of Lipinski's rule of five were calculated 
Mixed isatin with 3-(2-(aryl)hydrazono)acetylacetone Mn(II), Co(II) and Ni(II) complexes 539

using SwissADME web (http://swissadme.ch/index.php\#undefined). The computed molecular properties are shown in Table 2.

Table 1. Antibacterial activities of the mixed ligand metal complexes 1-9.

\begin{tabular}{|l|c|c|c|c|}
\hline \multirow{2}{*}{ Complexes No. } & \multicolumn{3}{|c|}{ Inhibition zone diameter (IZ) in millimeters } \\
\cline { 2 - 5 } & \multicolumn{2}{|c|}{ Gram-negative } & \multicolumn{2}{c|}{ Gram-positive } \\
\cline { 2 - 5 } & E. coli & P. aeruginosa & B. subtilis & S. aureus \\
\hline Complex 1 & 9 & 10 & 10 & 9 \\
\hline Complex 2 & 11 & 11 & 10 & 11 \\
\hline Complex 3 & 13 & 13 & 12 & 12 \\
\hline Complex 4 & 13 & 12 & 12 & 12 \\
\hline Complex 5 & 12 & 13 & 13 & 13 \\
\hline Complex 6 & 11 & 12 & 11 & 10 \\
\hline Complex 7 & 10 & 9 & 12 & 12 \\
\hline Complex 8 & 9 & 13 & 11 & 9 \\
\hline Complex 9 & 10 & 10 & 32 & 30 \\
\hline Tetracycline & 32 & 34 & & 11 \\
\hline
\end{tabular}

Compound: $>14 \mathrm{~mm}$, significant activity; 7-13 mm, moderate activity; $<7 \mathrm{~mm}$, weak activity.

Table 2. Lipinski's rule of five for the mixed ligand metal complexes 1-9.

\begin{tabular}{|c|c|c|c|c|c|}
\hline $\begin{array}{l}\text { The mixed ligand } \\
\text { metal complexes }\end{array}$ & $\mathrm{MW}^{\mathrm{a}}$ & $M \log \mathrm{P}^{\mathrm{b}}$ & $n \mathrm{HBA}^{\mathrm{c}}$ & $n \mathrm{HBD}^{\mathrm{d}}$ & $\mathrm{n}_{\text {violations }}$ \\
\hline Rule & $<500$ & $\leq 4.15$ & $\leq 10$ & $\leq 5$ & 0 \\
\hline Complex 1 & 444.06 & -0.86 & 7 & 3 & 0 \\
\hline Complex 2 & 444.30 & -0.86 & 7 & 3 & 0 \\
\hline Complex 3 & 440.31 & -0.86 & 7 & 3 & 0 \\
\hline Complex 4 & 478.51 & -0.36 & 7 & 3 & 0 \\
\hline Complex 5 & 478.75 & -0.36 & 7 & 3 & 0 \\
\hline Complex 6 & 474.75 & -0.36 & 7 & 3 & $1, \mathrm{MW}>500$ \\
\hline Complex 7 & 522.96 & -0.25 & 7 & 3 & $1, \mathrm{MW}>500$ \\
\hline Complex 8 & 523.20 & -0.25 & 7 & 3 & $1, \mathrm{MW}>500$ \\
\hline Complex 9 & 519.20 & -0.25 & 7 & 3 & 0 \\
\hline
\end{tabular}

${ }^{\mathrm{a}}$ Molecular weight; ${ }^{\mathrm{b}}$ Calculated lipophillicity $\left(M \log P_{\mathrm{o} / \mathrm{w}}\right)$; ${ }^{\mathrm{c}}$ Number of hydrogen bond acceptor; ${ }^{\mathrm{d}}$ Number of hydrogen bond donor; ${ }^{e}$ Violations from Lipinski's Rule.

From Table 2, all the mixed ligand metal complexes agreement with Lipinski's rule of five, where the metal complexes 1-6 in the range of the Lipinski's rule (MW $\leq 500$, MLogP $\leq$ $4.15, n H B A \leq 10$ and $n H B D \leq 5$ ) expected the three metal complexes 7-9, where the molecular weight of the complexes 7-9 more than 500 .

\section{CONCLUSION}

In conclusion, we have synthesized the $\mathrm{Mn}(\mathrm{II}), \mathrm{Co}(\mathrm{II})$ and $\mathrm{Ni}(\mathrm{II})$ complexes of mixed isatin with 3-(2-(aryl)hydrazono)acetylacetone 1-9. All the mixed ligand complexes were screened for their in vitro antibacterial activity. The evaluation showed that, among the tested complexes, the five complexes 3, 4, 5, 6 and $\mathbf{8}$ were the most active complexes against a panel of pathogenic tested organisms. Also, the mixed ligand metal complexes 1-6 agreement with Lipinski's rule of five.

\section{ACKNOWLEDGMENT}


The author wishes to express their thanks to the National Research Centre, Dokki, Cairo, Egypt for the facilities provided.

\section{REFERENCES}

1. Sakr, S.H.; Elshafie, H.S.; Camele, I.; Sadeek,S.A. Synthesis, spectroscopic, and biological studies of mixed ligand complexes of gemifloxacin and glycine with $\mathrm{Zn}(\mathrm{II}), \mathrm{Sn}(\mathrm{II})$, and Ce(III). Molecules 2018, 23, 1182.

2. Omar, M.M.; Abd El-Halim, H.F.; Khalil, E.A.M. Synthesis, characterization, and biological and anticancer studies of mixed ligand complexes with Schiff base and 2,2'-bipyridine. Appl. Organometal. Chem. 2017, 31, e3724.

3. Xu, Z.; Zhao, S.-J.; Lv, Z.-S.; Gao, F.; Wang, Y.; Zhang, F.; Bai, L.; Deng, J.-L. Fluoroquinolone-isatin hybrids and their biological activities. Eur. J. Med. Chem. 2019, 162, 396-406.

4. Guo, H. Isatin derivatives and their anti-bacterial activities. Eur. J. Med. Chem. 2019, 164, 678-688.

5. Hou, Y.; Shang, C.; Wang, H.; Yun, J. Isatin-azole hybrids and their anticancer activities. Arch. Pharm. Chem. Life Sci. 2020, 353, 1900272.

6. Hassan, M.; Ghaffari, R.; Sardari, S.; Farahani, Y.F.; Mohebbi, S. Discovery of novel isatinbased thiosemicarbazones: Synthesis, antibacterial, antifungal, and antimycobacterial screening. Res. Pharm. Sci. 2020, 15, 281-290.

7. Reddy, S.S.; Pallela, R.; Kim, D.-M.; Won, M.-S.; Shim, Y.-B. Synthesis and evaluation of the cytotoxic activities of some isatin derivatives. Chem. Pharm. Bull. 2013, 61, 1105-1113.

8. El-Naggar, M.; Eldehna, W.M.; Almahli, H.; Elgez, A.; Fares, M.; Elaasser, M.M.; AbdelAziz, H.A. Novel thiazolidinone/thiazolo[3,2-a]benzimidazolone-isatin conjugates as apoptotic anti-proliferative agents towards breast cancer: One-pot synthesis and in vitro biological evaluation. Molecules 2018, 23, 1420;

9. Abdel-aziz, H.A.; Eldehna, W.M.; Keeton, A.B.; Piazza, G.A.; Kadi, A.A.; Attwa, M.W.; Abdelhameed, A.S.; Attia M.I. Isatin-benzoazine molecular hybrids as potential antiproliferative agents: Synthesis and in vitro pharmacological profiling. Drug Des. Dev. Ther. 2017, 11, 2333-2346.

10. Salah, B.A.; Kandil, A.T.; Abd El-Nasser, M.G. Synthesis, characterization, computational and biological activity of novel hydrazone complexes. J. Radiat. Res. Appl. Sci. 2019, 12, 383-392.

11. Cheriyan, M.; Mohanan, K. Synthesis and characterization of the complexes of cobalt(II), nickel(II), copper(II) and zinc(II) with 2-(2-carboxyphenylazo)-1,3-diketones. Asian J. Chem. 2007, 19, 2831-2838.

12. Alzaydi, K.M.; Saleh, T.S. 2-Aryl hydrazonopropanalpharmacophores as potent cytotoxicagents against human hepatocellular carcinoma cell line. Med. Chem. Res. 2020, 29, 199-205.

13. Elsherif, M.A.; Hassan, A.S.; Moustafa, G.O.; Awad, H.M.; Morsy, N.M. Antimicrobial evaluation and molecular properties prediction of pyrazolines incorporating benzofuran and pyrazole moieties. J. Appl. Pharm. Sci. 2020, 10, 37-43.

14. Hassan, A.S.; Askar, A.A.; Nossier, E.S.; Naglah, A.M.; Moustafa, G.O.; Al-Omar, M.A. Antibacterial evaluation, in silico characters and molecular docking of Schiff bases derived from 5-aminopyrazoles. Molecules 2019, 24, 3130.

15. Khatab, T.K.; Hassan, A.S.; Hafez, T.S. $\mathrm{V}_{2} \mathrm{O}_{5} / \mathrm{SiO}_{2}$ as an efficient catalyst in the synthesis of 5-aminopyrazole derivatives under solvent free condition. Bull. Chem. Soc. Ethiop. 2019, $33,135-142$.

16. Hassan, A.S.; Moustafa, G.O.; Askar, A.A.; Naglah, A.M.; Al-Omar, M.A. Synthesis and antibacterial evaluation of fused pyrazoles and Schiff bases. Synth. Commun. 2018, 48, 2761-2772. 
Mixed isatin with 3-(2-(aryl)hydrazono)acetylacetone $\mathrm{Mn}(\mathrm{II}), \mathrm{Co}(\mathrm{II})$ and Ni(II) complexes 541

17. Magd-El-Din, A.A.; Mousa, H.A.; Labib, A.A.; Hassan, A.S.; Abd El-All, A.S.; Ali, M.M.; El-Rashedy, A.A.; El-Desoky, A.H. Benzimidazole-Schiff bases and their complexes: Synthesis, anticancer activity and molecular modeling as Aurora kinase inhibitor. $Z$. Naturforsch. C 2018, 73, 465-478.

18. Hassan, A.S.; Hafez, T.S. Antimicrobial activities of ferrocenyl complexes: A review. $J$. App. Pharm. Sci. 2018, 8, 156-165.

19. El-Naggar, M.; Hassan, A.S.; Awad, H.M.; Mady, M.F. Design, synthesis and antitumor evaluation of novel pyrazolopyrimidines and pyrazoloquinazolines. Molecules 2018, 23, 1249.

20. Hassan, A.S.; Awad, H.M.; Magd-El-Din, A.A.; Hafez, T.S. Synthesis and in vitro antitumor evaluation of novel Schiff bases. Med. Chem. Res. 2018, 27, 915-927.

21. Hassan, A.S.; Moustafa, G.O.; Awad, H.M. Synthesis and in vitro anticancer activity of pyrazolo[1,5-a]pyrimidines and pyrazolo[3,4-d][1,2,3]triazines. Synth. Commun. 2017, 47, 1963-1972.

22. Hassan, A.S.; Hafez, T.S.; Ali, M.M.; Khatab, T.K. Design, synthesis and cytotoxic activity of some new pyrazolines bearing benzofuran and pyrazole moieties. Res. J. Pharm. Biol. Chem. Sci. 2016, 7, 417-429.

23. Abd El-All, A.S.; Hassan, A.S.; Osman, S.A.; Yosef, H.A.A.; Abdel-Hady, W.H.; ElHashash, M.A.; Atta-Allah, S.R.; Ali, M.M.; El Rashedy, A.A. Synthesis, characterization and biological evaluation of new fused triazine derivatives based on 6-methyl-3-thioxo1,2,4-triazin-5-one. Acta Pol. Pharm. 2016, 73, 79-92.

24. Hassan, A.S.; Hafez, T.S.; Osman, S.A.; Ali, M.M. Synthesis and in vitro cytotoxic activity of novel pyrazolo[1,5-a]pyrimidines and related Schiff bases. Turk. J. Chem. 2015, 39, 1102-1113.

25. Hassan, A.S.; Osman, S.A.; Hafez, T.S. 5-Phenyl-2-furaldehyde: synthesis, reactions and biological activities. Egypt. J. Chem. 2015, 58, 113-139.

26. Helal, M.H.; Elgemeie, G.H.; El-Kashouti, M.A.; ElMolla, M.M.; Elsayad, H.S.; Ahmed, K.A. Synthesis of new 5-arylazo-3-cyano-4,6-dimethyl-2-acetylchloridethiopyridine reactive disperse dyes and their applications in textile printing. Pigm. Resin Technol. 2008, 37, 234-242.

27. Mijin, D.; Uscumlić, G.; Perisic-Janjić, N.; Trkulja, I.; Radetić, M.; Jovancić, P. Synthesis, properties and colour assessment of some new 5-(3- and 4-substituted phenylazo)-4,6dimethyl-3-cyano-2-pyridones. J. Serb. Chem. Soc. 2006, 71, 435-444.

28. Mahmudov, K.T.; Maharramov, A.M.; Aliyeva, R.A.; Aliyev, I.A.; Askerov, R.K.; Batmaz, R.; Kopylovich, M.N.; Pombeiro, A.J.L. 3-(Para-substituted phenylhydrazo)pentane-2,4diones: Physicochemical and solvatochromic properties. J. Photochem. Photobiol. A 2011, 219, 159-165.

29. Osman, S.A.; Mousa, H.A.; Yosef, H.A.A.; Hafez, T.S.; El-Sawy, A.A.; Abdallah, M.M.; Hassan, A.S. Synthesis, characterization and cytotoxicity of mixed ligand $\mathrm{Mn}(\mathrm{II}), \mathrm{Co}(\mathrm{II})$ and Ni(II) complexes. J. Serb. Chem. Soc. 2014, 79, 953-964.

30. Bauer, A.W.; Kirby, W.W.M.; Sherris, J.C.; Turck, M. Antibiotic susceptibility testing by a standardized single disc method. Am. J. Clin. Pathol. 1966, 45, 493-496.

31. Lipinski, C.A.; Lombardo, F.; Dominy, B.W.; Feeney, P.J. Experimental and computational approaches to estimate solubility in drug discovery and development settings. Adv. Drug Delivery Rev. 2001, 46, 3-26.

32. Lipinski, C.A. Lead- and drug-like compounds: the rule-of-five revolution. Drug Discovery Today: Technol. 2004, 1, 337-341. 